\title{
Prioritizing health services research: an economic perspective
}

\author{
Afschin Gandjour ${ }^{1}$
}

Received: 12 December 2015/ Accepted: 19 January 2016/Published online: 5 February 2016

(C) Springer-Verlag Berlin Heidelberg 2016

\begin{abstract}
Introduction Given limited resources policymakers need to decide about how much and in what areas of health services research (HSR) to invest. The purpose of this study is to provide guidance for priority setting of HSR projects based on economic theory.

Methods The conceptual analysis starts from the premise that competition in health care is valuable-a position that seems to predominate among Western policymakers. The principle of competition rests on economic theory and, in particular, its branch of welfare economics.

Results Based on economic theory, the role of HSR is to detect and alleviate information asymmetry, negative externalities, and harm caused by competition and inappropriate incentives for competition. A hierarchy of HSR projects is provided; following the ethical principle of harm ('do not harm'), the detection and prevention of harm would receive highest priority among HSR projects.

Conclusions Agreeing that competition is valuable in achieving efficiency and quality of care (and therefore agreeing to the assumptions of economic theory) implies accepting the role of HSR in detecting market failure and the HSR hierarchy as suggested. Disagreement would require an alternative coherent concept of improving efficiency and quality of care.
\end{abstract}

Keywords Health services research · Priority setting · Economics · Harm

Afschin Gandjour

a.gandjour@fs.de

1 Frankfurt School of Finance and Management, Sonnemannstr. 9-11, 60314 Frankfurt am Main, Germany

\section{Introduction}

In many industrialized countries, policymakers are divided about approaches to improve efficiency and quality of health care. On the one hand, policymakers often see competition among insurers and providers as a means to achieving these goals. On the other hand, they fund health services research (HSR), which ultimately also aims at improving efficiency and quality of health care (according to Bowling [2] the goal is to "produce reliable and valid research data on which to base appropriate, effective, costeffective, efficient and acceptable health services"). Given limited resources, however, policymakers need to decide about how much and in what areas of HSR to invest. In the United States, e.g., HSR currently receives $\$ 5.0$ billion per annum [7].

The purpose of this article is to provide guidance for priority setting of HSR projects based on economic theory (independent of a particular setting or country). It starts from the premise that competition in health care is valuable-a position that seems to predominate among Western policymakers. While the author does not hold a particular view on competition and its merits in health care, he discusses its implications for HSR when policymakers do have a favorable opinion.

\section{Theoretical framework}

The principle of competition rests on economic theory and, in particular, its branch of welfare economics. The latter suggests that competitive markets lead to efficiency and optimal outcomes. That is, markets are assumed to solve resource allocation problems including the question of how much and where to invest in health care in order to improve 
the efficiency and quality of care. In this framework regulation is only needed to ensure competition and reduce or eliminate 'market failure'. Market failure can be the result of harm, negative externalities, and information asymmetry. We define the terms as follows:

- Harm or, strictly speaking, net harm is the negative difference between health benefit and harm.

- Negative externality is an adverse impact on bystanders resulting from individual activity [6]. When bystanders are defined as insurees or taxpayers (depending on who finances the health care system), the adverse impact is an increase in insurance premiums or taxes.

- Information asymmetry is defined as a situation where patients have inadequate information and experience concerning their health problem and the quality of providers. Information asymmetry thus violates a requirement for making informed choices in markets.

According to the economic framework, the role of HSR would be to detect and alleviate information asymmetry, negative externalities, and harm to ensure competition, limit its undesirable consequences, and change inappropriate incentives. That is, competition as a means of improving efficiency and quality of care is insufficient because of market failure and would need to be complemented by HSR. As a word of caution, not every type of market failure falls within the domain of HSR, e.g., moral hazard.

To further describe specific causes of negative externalities and harm in competitive markets, we revert to the categorization of quality-of-care problems in underuse, overuse, and misuse [3]. Underuse is the failure to provide a healthcare service when it would have produced a favorable outcome for a patient. Overuse occurs when a health-care service is provided under circumstances in which its potential for harm exceeds the possible benefit (e.g., prescription of antibiotics for upper respiratory infections which are caused by a virus and are not effectively treated with antibiotics). And misuse occurs when an appropriate service has been selected, but a preventable complication occurs and a patient does not receive the full potential benefit of the service [4]. As a word of caution, definitions overlap and are not uniform (compare, e.g., the German Sachverständigenrat [8]).

Based on the above definitions, all quality problems as well as process inefficiency (e.g., waiting times or double work) may be sources of negative externalities. Misuse and overuse may also be sources of harm to patients.

\section{HSR hierarchy}

Following the ethical principle of harm ('do not harm'), the detection and prevention of harm receives highest priority among HSR projects (see Table 1). Research on misuse and
Table 1 Priority areas for publicly funded health services research

1. Detection and reduction of harm

2. Detection and reduction of negative externalities

3. Detection and reduction of information asymmetry

4. Detection/reduction of quality-of-care deficits and process inefficiency without harm or negative externalities

5. Detection of regional and international variation in health care

6. Evaluation of general health, mortality, quality of life, wellbeing, and spending

overuse is therefore prioritized. The same priority is given to research on underuse of acute services with immediate health benefits (e.g., underuse of cardiopulmonary resuscitation drugs). The reason is that this type of underuse results in immediate health deficits, similar to misuse and overuse with net harm. Note that harm may not only be the result of competition but also of poor regulation (e.g., insufficient educational requirements for physicians). The role of HSR would be to investigate harm regardless of whether it is caused by competition or regulation of competition.

Second on the list is research on the detection and elimination of negative externalities for insurees or taxpayers. This type of research offers opportunities for a net saving to the health care system. Judged by this criterion, the detection of overuse, misuse, and process inefficiency receives priority. A well-known example of overuse resulting from competition among providers is a medical arms race. Hospitals engage in a medical arms race when they spend unnecessarily on items such as cosmetic quality improvements, cost-enhancing technologies, and duplicate facilities as a way of attracting more physicians and patients [1]. Yet, overuse and process inefficiency may not cause a negative externality for insurees or taxpayers if they are borne by providers reimbursed by a lump-sum payment (e.g., diagnosis-related groups). On the other hand, underuse of health services may also impose a negative externality if the underused service leads to health system's savings. Before conducting research on underuse, it is therefore necessary to conduct a formal cost-effectiveness analysis (CEA) of the underused service in order to evaluate the potential for savings. The same holds for quality improvement interventions that address underuse (as an example for such evaluation see Gandjour [5]).

Third on the list is the detection and reduction of information asymmetry. It is ranked below the detection and reduction of harm and negative externalities simply because the link between better patient information and improvement in clinical or economic outcomes is less clear.

Forth ranks research on quality-of-care deficits and process inefficiency which do not cause harm or negative externalities for insurees or taxpayers. This includes obvious cases such as research on overuse and process 
inefficiency borne by the provider. A perhaps more controversial case would be research on the underuse of services which do not result in savings including the case of zero savings. One may counter argue that it would be unethical to give lower priority to this type of research as opposed to research on underuse which does result in savings. Yet, resources for HSR are scarce and policymakers who believe in competition and markets should rely on self-regulatory forces of markets as this is the operating framework to begin with. That is, in the case where underuse is related to drugs or medical devices, manufacturers should have an incentive for funding the detection of underuse because higher future sales are expected. In support of this argument, there is, in fact, a growing role of the 'industry' in funding HSR in the US [7]. When services are not 'manufactured' but are based on human interaction (such as psychotherapy), medical professional organizations should be trusted to fund research on underuse. Unlike the case of overuse, medical professional organizations should have a financial incentive because the typical 'market' response to undersupply is a fee increase.

Further down the list is research on regional and international variation in health care (including so-called smallarea analyses). The reason is that such research may not provide direct recommendation for improving the quality of care and changing inappropriate incentives. Hence, it needs to be followed by an investigation of quality-of-care problems. Take the example of international variation in the number of coronary artery bypass graft surgeries (CABGs) per 1000 patients with coronary heart disease. Showing that country A is on top of the list does not necessarily mean that the degree of overuse is highest too. Instead, it may be that CABG is underused in other countries. Therefore, we need to investigate both overuse and underuse in the various countries. But then we could also start investigating overuse and underuse without a study on international variation. Only when interventions can be clearly labeled as overuse, underuse, or misuse (unlike the CABG case), this type of research would lead to direct recommendations.

At the bottom of the priority list are evaluations of general health, mortality, quality of life, well-being, and spending (including those on regional and international variation). As these studies are not intervention-specific and capture factors outside the health care system, they must always be followed by an investigation of overuse, misuse, underuse, and process inefficiency.

\section{Conclusions}

In summary, I would like to highlight a few points. While it seems obvious that patient safety and the detection of harm should receive priority, it has not been established that funding the detection and reduction of underuse should be preceded by an assessment of the cost-effectiveness of underused services. Only if CEAs demonstrates savings, public funds for reducing underuse should be made available; otherwise, and in view of limited public resources, we should trust the market to correct underuse. The criteria provided in this paper for funding HSR are more specific than the traditional ones such as disease prevalence, size of quality deficit, potential for quality improvement, and high treatment costs.

Policymakers and readers who agree that competition is valuable in achieving efficiency and quality of care (and therefore implicitly accept the assumptions of economic theory) would also, to a large extent, need to agree to the role of HSR in detecting market failure and the HSR hierarchy as suggested. Policymakers and readers who do not agree would need to provide an alternative coherent concept of improving efficiency and quality of care. Also, moving low-priority areas up the list would need to be discussed in view of limited resources for health care and HSR.

Acknowledgments The author would like to thank participants at a meeting of the Health Economics Group of the German Society for Health Services Research (2015) for very valuable comments on an earlier draft.

\section{Compliance with ethical standards}

Funding This research received no specific grant from any funding agency in the public, commercial or not-for-profit sectors.

\section{References}

1. Bates, L.J., Mukherjee, K., Santerre, R.E.: Market structure and technical efficiency in the hospital services industry: a DEA approach. Med Care Res Rev. 63(4), 499-524 (2006)

2. Bowling, A.: Research Methods in Health: Investigating Health and Health Services. Open University Press, Buckingham (1997)

3. Chassin, M.R.: Practice guidelines: best hope for quality improvement in the 1990s. J. Occup. Environ. Med. 32(12), 1199-1206 (1990)

4. Donaldson, M.S.: Institute of Medicine. Statement on Quality of Care. National Academies Press, Washington, DC (1998)

5. Gandjour, A.: A model to predict the cost-effectiveness of disease management programs. Health Econ. 19(6), 697-715 (2010)

6. Mankiw, N.G.: Principles of Economics. South-Western Cengage Learning, Mason (2008)

7. Moses 3rd, H., Matheson, D.H., Cairns-Smith, S., George, B.P., Palisch, C., Dorsey, E.R.: The anatomy of medical research: US and international comparisons. JAMA 313(2), 174-189 (2015)

8. Sachverständigenrat für die Konzertierte Aktion im Gesundheitswesen. Bedarfsgerechtigkeit und Wirtschaftlichkeit. Gutachten 2000/2001, Bd III. Bonn: SVR (2001) 